\section{Asthmaschulung in Deutschland}

\section{Erstes einheitliches Programm}

\author{
In der Therapie des Asthma hat die Patientenschulung einen hohen \\ Stellenwert. Mit dem NASA-Programm wird nun erstmalig in \\ Deutschland eine einheitliche, strukturierte, ambulante Schulung \\ angeboten. Ziel dieser von führenden Pneumologenverbänden ge- \\ tragenen Initiative ist eine grundlegende Verhaltensänderung der \\ Patienten, die ihre Krankheit eigenverantwortlich managen sollen.
}

W ie für jede chronische Krankheit gilt auch für das Asthma bronchiale: Die Therapie kann nur dann erfolgreich sein, wenn die Patienten aktiv mitarbeiten an der Bewältigung ihrer Erkrankung. Diese Erkenntnis hat sich bereits mit den Anfängen der Patientenschulung im Jahr 1984 durchgesetzt und zu einer Vielzahl von Schulungsprogrammen und -Initiativen in Deutschland geführt.

\section{Pneumologenverbände einig}

Doch ein Manko gibt es noch: Es existierte im ambulanten Bereich kein bundesweit etabliertes, standardisiertes und evaluiertes Schulungsprogramm für erwachsene Asthmatiker. Deshalb haben die Deutsche Atemwegsliga, der Deutsche Berufsverband der Pneumologen und die Sektion Prävention und Rehabilitation der Deutschen Gesellschaft für Pneumologie gemeinsam das „Nationale ambulante Schulungsprogramm für erwachsene Asthmatiker" (NASA) aus der Taufe gehoben, so $\mathrm{H}$. Worth, Fürth.

Die validierte Fassung steht jetzt für die Umsetzung in der Praxis bereit (s. Kasten). Der Nutzen des neuen Programms konnte bereits in randomisierten Prüfungen belegt werden: Die geschulten Asthmatiker hatten ihre Erkrankung deutlich besser „im Griff“ als nicht geschulte Kontrollasthmatiker.

\section{Schulungskärtchen für die Ärzte}

Schulungswilligen Ärzten bieten die Organisationen intensive Unterstützung an. Sie erhalten das strukturierte Programm auf vorgefertigten Unterrichtskarten zusammen mit Bildmateri- al und einem pädagogischen Leitfaden. Die Ausbildung der Ärzte erfolgt in so genannten Train-the-Trainer-Seminaren. Auffrischungskurse für Patienten und Lehrer dienen der Festigung eines dauerhaften Schulungserfolges.

\section{Knackpunkt: finanzieller Anreiz}

Die Voraussetzungen für ein flächendeckendes, einheitliches und evaluiertes Asthmaschulungsprogramm sind damit durch die führenden wissenschaftlichen Asthmaorganisationen in Deutschland geschaffen, die Schulungen der Praxisteams durch die Atemwegsliga gewährleistet.

\section{NASA - dem Asthma Paroli bieten}

Die Asthmapatienten sollen in den Kursen lernen,

- die Inhalationshilfen korrekt anzuwenden,

- sich im Notfall richtig zu verhalten, lichen Punkten der Schulung vorgesehen. Patientenschulung.

\section{NASA-Infos}

Informationen über das NASA-Programm sowie zu den Train-the-Trainer-Seminaren gibt die Deutsche Atemwegsliga e.V., Burgstraße 12, D-33175 Bad Lippspringe, Tel.: 05252 / 933615 , Fax: 05252 / 933616 , E-Mail: Atemwegsliga.Lippspringe@tonline.de oder

Atemwegsliga.U.Butt@t-online.de, Internet: www.atemwegsliga.de

Damit die Bemühungen aber auch wirklich den Asthmapatienten erreichen, fehlt zumindest in den meisten Bundesländern noch der entscheidende Anreiz für die Trainer - die finanzielle Vergütung -, bedauerte Worth. Lediglich Ärzte in Bayern erhalten seit 1999 eine angemessene Aufwandsentschädigung.

\section{Worth H}

Pressekonferenz der Deutschen Atemwegsliga im Rahmen des 32. Bad Reichenhaller Kolloquiums, Juni, 2000.

Das „Nationale ambulante Schulungsprogramm für erwachsene Asthmatiker“ (NASA) ist ein strukturiertes und evaluiertes Schulungsprogramm für den bundesweiten Einsatz in den Praxen niedergelassener Ärzte.

- die Symptome regelmäßig mit Hilfe des Peak-flow-Meters zu überwachen,

- eine kontrollierte Selbstmedikation durchzuführen, d.h. nach ärztlicher Verordnung die Dosis der Medikamente an den jeweiligen Asthmaschweregrad anzupassen,

- Atemtechniken für den Alltag und den Notfall zu erlernen sowie

- die Asthmaerkrankung und ihre Therapie zu verstehen.

Eine Schulungsgruppe umfasst vier bis acht erwachsene Asthmatiker, die das Wissen in sechs bis acht jeweils 6o-minütigen Unterrichtseinheiten vermittelt bekommen. Zur Aufrechterhaltung der Schulungseffekte sind „Refresherkurse“ mit den wesent-

Der Trainer oder das Trainerteam, in der Regel Arzt/Praxishelferin, sollte über ein Mindestmaß an pädagogischem Geschick verfügen - der Rest ist erlernbar durch die Teilnahme an einem Lehrverhaltenstraining, der konsequenten Nutzung des NASASchulungsprogramms und evtl. durch eine Hospitation bei einer bereits etablierten 